\title{
Simulación por el método de Monte Carlo para generar criterios de aceptación en el control de calidad de productos de construcción
}

\author{
The Monte Carlo methods for use as criteria generator in \\ construction products quality control
}

\author{
$\underline{\text { A. Garrido }}^{(*)}$, E.M. Conesa $a^{(*)}$
}

\section{RESUMEN}

En el Sector de la Construcción es habitual realizar operaciones de control de la calidad de aquellos productos o procesos prescritos por los reglamentos. Raramente, por tanto, se llevan a cabo operaciones de recepción de productos por pactos espontáneos entre las partes. Esto último no ocurre, fundamentalmente, porque los que toman decisiones empresariales o técnicas a este respecto no conocen las bases estadísticas del control de conformidad $y$, por tanto, no conocen cómo operar en consecuencia. Este artículo propone un método para que los agentes involucradas en la recepción de productos, una vez fijado el riesgo asociado al control estadístico que les corresponde, definan el criterio de aceptación más adecuado.

\section{3-20}

Palabras clave: Monte Carlo, control de calidad, simulación, riesgo del consumidor, curvas OC, estimación estadística.

\section{ANTECEDENTES DEL MÉTODO}

El método de Monte Carlo (1) es un procedimiento numérico que permite aproximar la resolución de expresiones matemáticas complejas con las que resulta o bien difícil, o bien imposible (especialmente en el ámbito
Informes de la Construcción Vol. 61, 515, 77-85, julio-septiembre 2009 ISSN: 0020-0883 elSSN: 1988-3234 doi: 10.3989/ic.09.025

\section{SUMMARY}

Quality Control in Building projects is a topic of technical specifications. Nevertheless, it is not usual that costumers ask for additional control out of de technical specifications. The reason is they don't know how to do or they consider it is a very difficult issue. This article proposes an easy way to resolve that question by an old and, to a certain extends, a young method: Monte Carlo simulator. This way, suppliers and its costumers (assessing by their advisers) have a powered tool to resolve they relationships by means of statistical method without special problems of understanding. A Method based in the consumer risk that allow establish a good control criteria.

Keywords: Monte Carlo, quality control, simulator, consumer risk, OC curve, statistic estimation.

de la estadística) encontrar resultados exactos. Al método se llegó en dos etapas. En la primera, los brillantes matemáticos racionalistas del siglo XVIII abordan la búsqueda de valores crecientemente precisos de número irracionales por distintas vías. Pero es George Louis Leclerc, conde de Buffón, un conocido

\footnotetext{
(*)Arquitecto Técnico. Escuela de Arquitectura e Ingeniería de Edificación. Universidad Politécnica de Cartagena (España).

${ }^{(* *)}$ Ingeniero Industrial. Escuela de Arquitectura e Ingeniería de Edificación. Universidad Politécnica de Cartagena (España). 
naturalista, botánico, matemático y cosmólogo francés quien en 1777 aborda un método experimental para la aproximación del número $\pi$, lo que supone un ingenioso modo de plantear la relación entre la realidad y las matemáticas. Buffón (Figura 1) demostró que dejando caer agujas sobre unas franjas cuya anchura fuera igual a la longitud de las agujas la probabilidad de que la aguja pase por las franjas es la indicada en la ecuación [1]:

$\mathrm{P}(\mathrm{a})=\frac{2}{\pi}$

De forma que, como se observa en la ecuación [2]:

$\pi=\frac{2 \mathrm{~N}}{\mathrm{n}}$

Siendo $\mathrm{N}$ el número total de intentos y $\mathrm{n}$ el número de veces que la aguja pasa por las franjas. Naturalmente se ha considerado, con una concepción frecuentista, que la probabilidad es la indicada en la ecuación [3]:

$$
\mathrm{P}(\mathrm{a})=\frac{\mathrm{n}}{\mathrm{N}}
$$

La segunda etapa se produce 180 años después, cuando Stanislaw Ulam en 1946 jugando a los solitarios durante su colaboración en el proyecto Manhattan en Los Álamos, concibió la idea de que fórmulas complejas sin solución aparente sobre difusión de neutrones podían ser aproximadas mediante el establecimiento de probabilidades a priori del fenómeno o, lo que significa lo mismo, darle forma probabilística al proceso para luego reproducirlo mediante repeticiones al azar. Para que el método fuera seguro se necesitaban muchas repeticiones y para eso vino en su ayuda Von Neuman que, un año después, ya tenía a punto el método y la herramienta: los ordenadores incipientes de la época.

\section{CONCEPTOS PRINCIPALES}

\subsection{Riesgo del Consumidor}

El riesgo del consumidor es un concepto introducido por Dodge y Romig en 1929

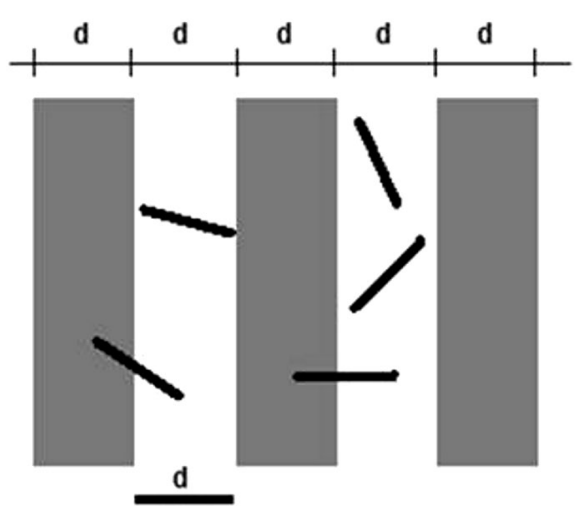

(3), normalmente notado como $\beta$, es, en el marco del control estadístico, la probabilidad de aceptar un lote de un producto cuando éste no es conforme. En términos más generales, es la probabilidad de aceptación cuando el nivel de calidad tiene un valor establecido por el plan de muestreo de aceptación como no satisfactorio (5). En rigor, es la probabilidad de aceptación de un lote cuando el producto es estrictamente conforme. Pero como una pequeña desviación respecto del valor especificado implica no conformidad se conviene en que la probabilidad de aceptar un lote no conforme sea igual a la de aceptar un lote en el que el parámetro de conformidad y el valor especificado sean iguales.

Esta probabilidad es complementaria a la unidad con el riesgo del suministrador, normalmente notado como $\alpha$. Tal y como indica la ecuación [4]:

$\alpha=1-\beta$

La ecuación [4] es válida para un producto estricto, en el sentido explicado más arriba.

El consumidor y el suministrador, pues, se reparte la incertidumbre asociada al hecho de que, en el control estadístico (2) (3) (10), las muestras son relativamente pequeñas y, por tanto, la función de aceptación que se considere es una variable aleatoria que proporciona la probabilidad de aceptación en función del cuantil del valor especificado. De hecho, el valor de $\beta$ viene dada por el área de la distribución de probabilidad del criterio de aceptación que queda a la derecha del valor especificado, si se trata de una característica que se considera favorable con valores mayores que el especificado.

Dada la definición de $\beta$ como probabilidad de aceptar un lote no conforme de un producto, es necesario establecer un procedimiento para su determinación. El más adecuado es el de considerar esta probabilidad justo para el caso en que el lote del producto es estrictamente conforme.

De hecho, se puede considerar que en el control de calidad la cuestión más importante, desde el propósito de este artículo, es establecer la distribución de probabilidad de la variable definida para operar con los valores resultantes del muestreo. Variable cuyos valores son comparados con el valor especificado para aceptar o rechazar el lote. Dada una función de aceptación determinada, su desplazamiento relativo al valor especificado se produce con la calidad relativa del producto proporcionando sucesivos valores de la probabilidad de aceptación $\mathrm{P}(\mathrm{A})$ mayo- 
res o menores que la que hemos definido como riesgo del consumidor. Las parejas de valores $\mathrm{P}(\mathrm{A}), \boldsymbol{\theta}$ (valor del parámetro de calidad escogido y a comparar con el valor especificado genérico $\theta_{\mathrm{LQ}}$ ) son coordenadas que unidas proporcionan una curva muy especial que denominamos Curva Operativa de Control o Curva Característica de Operación, según el vocabulario aceptado por la Norma UNE -ISO 3534-2:2008 (5). Las curvas Características de Operación indican el porcentaje de lotes que pueden ser aceptados, según los diferentes planes de muestreo para una calidad de proceso determinada. Se entiende por eficacia a la mayor o menor rapidez con que desciende la probabilidad de aceptación del producto ante una determinada desviación del valor especificado.

\subsection{La Curva Característica de Operación}

La Curva Característica de Operación (CO) proporciona una visión panorámica de la mayor o menor eficacia de un determinado criterio de aceptación. Se entiende por eficacia a la mayor o menor rapidez con que desciende la probabilidad de aceptación del producto ante una determinada desviación para peor del valor especificado. Es decir, la curva CO está compuesta por los pares de puntos $(\theta), P(A)$ calidad del producto), Probabilidad de Aceptación para un determinado plan de control o, lo que es lo mismo, para un determinado criterio de aceptación. Así, por ejemplo, un criterio de estructura general, como se indica en la ecuación [5]:

$\bar{x}_{N}-K S_{N} \geq V_{e}$

Tendrá tantas curvas CO como tamaños de la muestra o valores de K. Pero todas ellas pasarán por el punto cuyas coordenadas viene dadas por el valor de la característica de calidad elegida $(\theta)$ que se corresponda con el valor especificado (Ve) y el valor del Riesgo del Consumidor $(\beta)$. Valor que va implícito al tamaño de la muestra y al valor de Ko, en términos más generales, a la estructura del criterio de aceptación.

\subsection{Los criterios de aceptación o rechazo (A/R) en la normativa española}

Podemos suponer que la mayoría de las características de los materiales de construcción tienen una función de densidad regida por la función de la ecuación [6]:

$f(x)=\frac{1}{\sigma \sqrt{2 \pi}} e^{\frac{1}{2}\left(\frac{x-\mu}{\sigma}\right)^{2}}, x \in R_{N}$
Esta distribución se cumple en todo caso para la distribución de la suma de variables aleatorias con distribución distinta de la normal, como muestra el Teorema Central del Límite (9). Lo que es de aplicación a la distribución del valor medio de muestras procedentes de valores aleatorios. Es decir, por la distribución de densidad de la curva normal. Por eso, las funciones de aceptación han tomado formas idénticas o derivadas de dicha función normal, como se indica en la ecuación [7]:

$\bar{x} \pm 1,645 \sigma$

Fórmula que corresponde a valores de la variables asociados al cuantil 5/95\% según el signo negativo o positivo adoptado.

Pero también se adoptaron formas menos explícitas como la presentada en la fórmula [8]:

$\mathrm{x}_{1} \cdot \mathrm{K}_{\mathrm{N}}$

Por otra parte, al menos en lo referente al hormigón estructural, durante años el concepto que ha regido el control de calidad de los materiales ha sido el de estimación. Es decir, definida una propiedad por su valor característico, el problema del control consistía en comparar el valor especificado con una estimación verosímil del parámetro correspondiente a la propiedad controlada en la producción real de un determinado fabricante. El valor característico es aquel que representa de forma convencionalmente establecida (normalmente por los reglamentos) a todos los que potencialmente pueden darse en una población determinada. En este sentido la fórmula [7] proporciona valores característicos asociados al cuantil $5 \%$ y $95 \%$ (según la circunstancia de que se trate) expresada con el signo. De ahí deriva el que llamemos valor característico especificado a aquél respecto del cual se toman las decisiones, como expresa la definición de dicho valor en la Instrucción Española de Hormigón Estructural (6). Así, los criterios de aceptación tenían la forma de una estimación a partir de la desigualdad general indicada en la ecuación [9]:

estimación del valor real $\geq$ valor especificado [9]

Que tomaba para el hormigón formas como se observa en la ecuación [10]:

$f_{c \text {, real }} \geq f_{c k}$

Con la correlación consiguiente en el criterio de aceptación dado en la ecuación [11]:

$x_{1}+x_{2}-x_{3} \geq f_{c k}$ 
Siendo $x_{1}+x_{2}-x_{3}$ un estimador rígido de $\mathrm{f}_{\mathrm{c} \text {, real }}$

En otros casos, tomaba formas como la presentada en la ecuación [12]

$\mathrm{x}_{1} \cdot \mathrm{K}_{\mathrm{N}}>\mathrm{f}_{\mathrm{ck}}$

Siendo $\mathrm{x}_{1} \mathrm{~K}_{\mathrm{N}}$ una estimación de $\mathrm{f}_{\mathrm{c} \text {, real }}$ cuando $K_{N}$ tenía el valor adecuado para que la mediana de la distribución del estimador coincidiera con la resistencia real.

Hoy en día se ha preferido desvincular la función de aceptación de la estimación, que pasará a ser un caso particular: aquél en el que el riesgo del consumidor $\beta$ es igual al $50 \%$. Así ha procedido en la Instrucción Española de Hormigón Estructural (6) al establecer valores de $\beta$ menores del 50\% para determinadas situaciones, $\mathrm{o}$, como ocurre en el control de producción de productos como el cemento, en los que los valores centrales asociados a la esperanza matemática de la distribución se aleja notablemente del valor característico de la población, como muestra más adelante la figura 5 .

La consecuencia es la libertad de los actores para fijar las condiciones que asumen en su relación contractual. Es el caso de aquellas circunstancias en las que los reglamentos no proporcionan ninguna regla al respecto o que se desea modificar el riesgo del consumidor. ¿Qué hacer en esos casos? La respuesta es la deducción de la fórmula que proporciona la probabilidad de aceptación en función de la característica de calidad escogida (lo que no siempre es posible) o bien utilizar el método de Monte Carlo.

\section{EL MÉTODO DE MONTE CARLO Y LOS CRITERIOS A/R}

El método de Monte Carlo en la aplicación que proponemos en este artículo funciona mediante la función ALEATORIO de la hoja de cálculo EXCEL ${ }^{T M}$ de Microsoft (13) insertada en la ecuación del fenómeno que se pretende simular.

En nuestro caso se trata de generar valores de forma indefinida y aleatoria procedentes de una población cuya distribución de densidad se conoce. Dado que, habitualmente, la distribución normal es la que mejor explica el comportamiento de las variables físicas consideradas en la construcción, la ecuación [13] toma la forma:

$X=\mu+Z \sigma$

Siendo:

$\mathrm{X}$ un valor aleatorio de la característica considerada $\mu$ el valor de la media de la población de procedencia

$\sigma$ el valor de la desviación estándar de la población

Es decir, cada valor aleatorio es seleccionado al azar como resultado de aplicar esta fórmula con valores, igualmente aleatorios, de Z. Los valores aleatorios de Z se obtienen al aplicar la función ALEATORIO. Esta función proporciona espontáneamente un valor al azar comprendido entre 0 y 1 . Dado que las probabilidades en una distribución de densidad vienen representadas por el área que queda a la izquierda o a la derecha de un determinado cuantil, si consideramos adicionalmente que la función de $\operatorname{EXCEL}^{\circ}$, de las familia de las estadísticas, DISTR.NORM.ESTAND.INV () proporciona un valor de la variable tipificada $Z$ para cada valor del argumento comprendido entre 0 y 1 , tenemos la ecuación generadora [14] (10):

$X=\mu+$ DISTR.NORM.ESTAND.INV(ALEATORIO()). $\sigma \quad[14]$

En la que ALEATORIO () genera valores de probabilidad al azar y $Z$ es generada por DISTR.NORM.ESTAND.INV(ALEATORIO ()). De este modo, obtenemos tantos valores de $X$ como necesitemos para nuestro propósito.

Una vez generados los valores, en la forma que simule más apropiadamente nuestro proceso de control, se está en condiciones de organizar los datos simulando las condiciones de recepción de un material. Es decir, lotes, muestras, ensayos. Estamos ahora en condiciones de representarnos las cosas como si las matemáticas no existieran, vana pretensión pues partimos de una fórmula que expresa precisamente la estructura de los datos, pero nuestra pretensión sería correcta si los datos los obtuviésemos tras prolongadas sesiones experimentales en las que consiguiéramos un número de datos suficientemente alto como para asegurar que las conclusiones son válidas y, de esta forma, adquirir conocimiento sobre la estructura de la realidad.

Como veremos en el ejemplo final, procedemos a aplicar a los datos ordenados conforme a un determinado plan de control el criterio de aceptación que queramos poner a prueba en un número suficientemente alto de lotes. Por ejemplo 50.000 veces.

De esta forma quedan corregidas las oscilaciones propias del tamaño de la muestra y se obtienen valores medios suficientemente estables. 


\subsection{Aplicación a planes no reglados}

Vamos a ver con un ejemplo cómo funciona el método. Naturalmente vamos a proceder del modo que le es tan caro a la estadística. Es decir, usando un criterio de aceptación del que se sabe todo para que sirva de contraste con los resultados obtenidos. El criterio en cuestión pasará por ser un diseño nuestro justificado debidamente por la naturaleza de nuestro plan de control, que viene condicionado por razones como el tamaño de la muestra, la confianza en la coherencia interna del sistema de producción, etc. El criterio en cuestión es el expresado en la ecuación [15], tomado de la Instrucción Española de Hormigón Estructural (6):

$\bar{x}_{N}-K \cdot r_{N} \geq f_{c k}$

Siendo:

$\overline{\mathrm{X}}_{\mathrm{N}}$ : el valor medio de la muestra

$\mathrm{K}$ : un coeficiente modulador del efecto del criterio o constante de aceptabilidad

$r_{N}$ : el recorrido de la muestra como $\left(x_{N}-x_{1}\right)$ $\mathrm{f}_{\mathrm{ck}}$ : el valor especificado

La fórmula [15] presenta uno de los criterios de aceptación posibles en el que se ha escogido el recorrido de la muestra en vez de (por ejemplo) el valor s de la desviación estándar muestral suponiendo un caso de número bajo de muestras para tomar decisiones sobre el lote.

Aunque se trata de un criterio para la resistencia del hormigón estructural, procederemos como si sirviera (lo que no está muy alejado de la realidad) para cualquier otro material y cualquier otra característica.

La estructura del criterio nos habla de una familia de criterios, pues habrá tantos criterios concretos como valores de $\mathrm{K}$ podamos imaginar. Los valores de $x^{-}{ }_{N} y$ de $r_{N}$ se producen del azar dentro de los límites de la estructura estadística de la producción. Si no existiese un valor como $\mathrm{K}$ el criterio actuaría de forma rígida y las probabilidades de aceptación para cada nivel de calidad del producto serían igualmente fijas y determinadas. Sin embargo, con el coeficiente $\mathrm{K}$ es posible modificar la probabilidad de aceptación y, por tanto, la principal de ellas, la que corresponde a la situación de producto conforme o estricto que hemos denominado supra como el riesgo del consumidor $(\beta)$.

Es importante distinguir entre el criterio de conformidad y el de aceptación. El primero es la condición que nos permite aceptar un lote cuando tenemos toda la información y el criterio de aceptación es el que nos permite, en condiciones aceptadas de incertidumbre, aceptar el lote cuando sólo contamos con la información de la muestra. En este caso consideramos como tal criterio de conformidad el expresado en la ecuación [16]:

$f_{c \text {, real }} \geq f_{c k}$

Es decir el producto es conforme cuando se cumple esta condición y no lo es cuando no se cumple. Se considera producto estricto a aquel que cumple la condición de la ecuación [17]:

$\mathrm{f}_{\mathrm{c} \text {, real }}=\mathrm{f}_{\mathrm{ck}}$

Este valor de $\mathrm{f}_{c \text {, real }}$ es el que figurará directamente en las abscisas de la curva OC o bien representado por otro parámetro de calidad directamente relacionada con él, como puede ser la relación $f_{c \text {, real }} / f_{c k}$ o el área que queda a la izquierda de $f_{c k}$ en la distribución de densidad de la característica considerada del producto, etc.

Para aplicar el método de Monte Carlo procedemos del siguiente modo:

1. Aceptamos que: $X=\mu+Z \sigma$

2. Traducimos a las funciones de EXCEL

$X=m+D I S T R . N O R M . E S T A N D . I N V(A L E A T O R I O()) \cdot \sigma \quad[19]$

3. Estructuramos la recepción en lotes que se juzgan con muestras de tamaño $\mathrm{N}=3$

4. Fijamos un valor especificado de 30 unidades

5. Fijamos un valor de la desviación estándar de 3 unidades

6. Fijamos, en principio un valor de $f_{c \text {, real }}=f_{c k}$ 7. Generamos 50.000 grupos de 3 valores (lotes)

8. Definimos exploratoriamente el criterio de aceptación $\bar{x}_{N}-K \cdot r_{N} \geq f_{c k}$ 9. Fijamos un valor exploratorio para $K=1,5$ 10. El criterio queda así: $x_{N}-1,5 \cdot r_{N}>f_{c k}$ 11. Aplicamos 9 a los 50.000 lotes

12. Damos un valor de 1 cuando se cumple 9 y 0 cuando no se cumple 8

13. Sumamos las columnas de unos y ceros y dividimos por 50.000, siendo el resultado de la operación la frecuencia relativa de aceptaciones en 50.000 casos. 14. Traducimos la frecuencia relativa en probabilidad y ya tenemos la probabilidad de aceptar un lote no conforme cuando se trata de un producto estricto. Es decir, tenemos el riesgo del consumidor de nuestro criterio de aceptación ( $\beta$ ).

15. Analizamos el valor de $\beta$ y comprobamos que es 0,29. Es decir, el $29 \%$ de los 50.000 lotes han sido aceptados. Como se trata de un producto estricto, esta probabilidad es el riesgo del consumidor. 

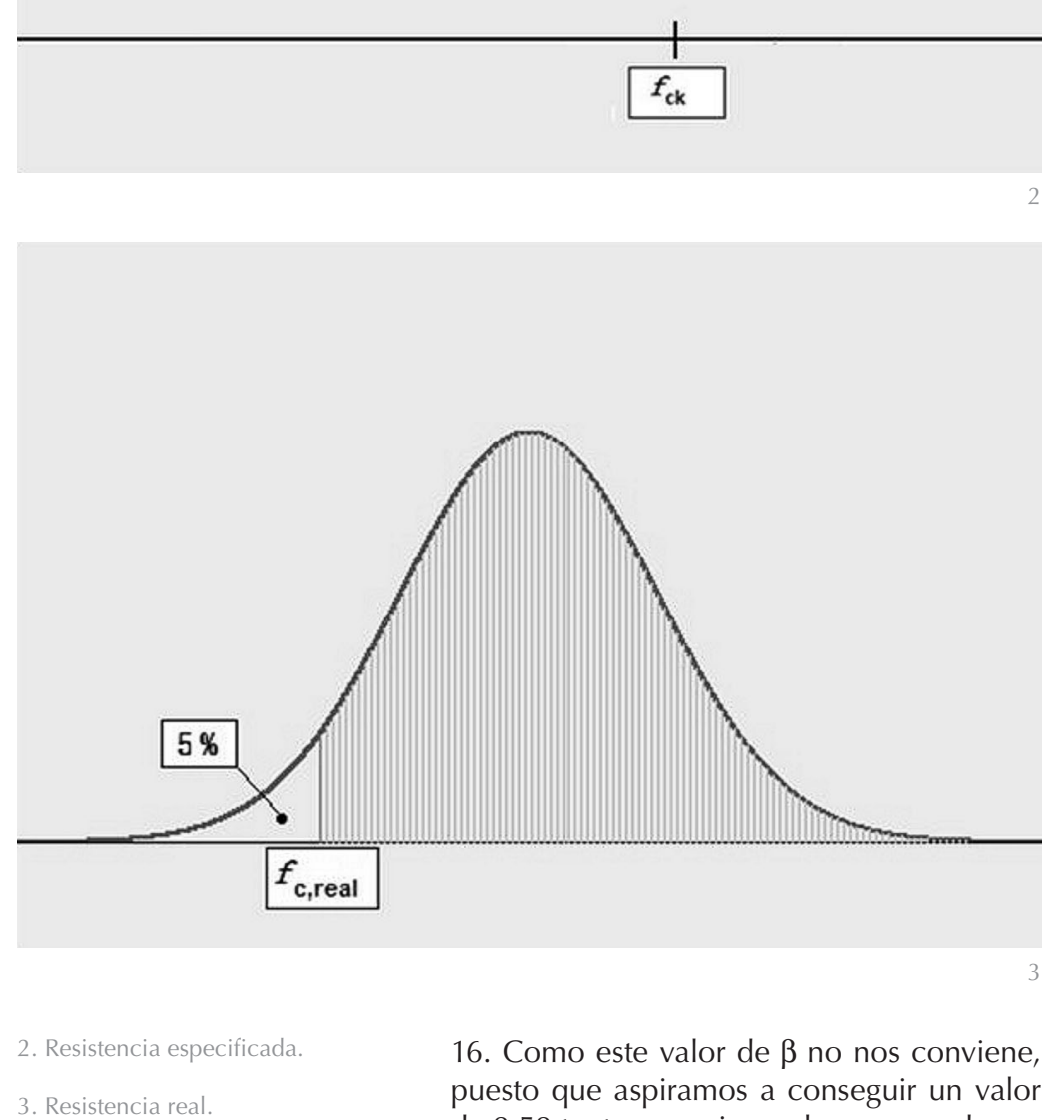

16. Como este valor de $\beta$ no nos conviene, puesto que aspiramos a conseguir un valor de 0,50 tanteamos si su valor crece o decrece con el aumento de $\mathrm{K}$.

17. Comprobado que $\beta$ aumenta cuando decrece $\mathrm{K}$, procedemos a rápidos tanteos y en unos pocos minutos se comprueba que el valor de $\beta$ buscado se obtiene cuando $K$ $=1,019$.

Como hemos tomado como referencia un criterio de aceptación que sí está regulado, comprobamos en la tabla 86.5.4.3.b de la Instrucción Española de Hormigón Estructural (6) que el valor de $\mathrm{K}$ para el riesgo del consumidor $\beta=0,50$ es, precisamente 1,02. Naturalmente, si queremos conocer qué criterio se corresponde con un valor de $\beta=0,05$, si ese fuera nuestro interés, bastaría con tantear valores de $\mathrm{K}$ mayores que la última referencia que teníamos $(1,5)$ y con rapidez obtenemos el valor correspondiente, que es un número redondo, $\mathrm{K}=4$.

Para visualizar el proceso de simulación, el concepto de Riesgo del Consumidor y efecto de $\mathrm{K}$ en los criterios de aceptación proporcionamos una secuencia de imágenes. La Figura 2 muestra el carácter de dato convencional del valor especificado por el autor del proyecto. No va asociado a una distribución y se fija como referente para la aceptación o rechazo de los productos.

Tradicionalmente el valor especificado (Figura 2) ha sido comparado con valores procedentes de acciones experimentales no siempre bien descritas y, en muchos casos, asociados al valor medio de una muestra sin relación alguna con la dispersión de valores como consecuencia de la propia variabilidad de la población de procedencia y de la variabilidad asociada al tamaño de la muestra.

La Figura 2 expresa el carácter determinista del valor especificado. Valor que servirá de referencia para determinar si el lote es aceptado o no. Con lo que se constituye en frontera entre los valores de la función de aceptación.

En la aplicación reciente de criterios de aceptación, toda la variabilidad es considerada fundamental e incluso, en reglamentos recientes, se ha optado por exigir la certificación con respaldo oficial de los parámetros de variabilidad, tales como la desviación estándar u otros.

En el caso de situaciones contractuales sin respaldo reglamentario el método de Monte Carlo se erige en una opción de fácil aplicación y, sobre todo, de fácil comprensión por todos los agentes involucrados. Veamos ahora la secuencia implícita. La Figura 3 muestra la distribución normal $\mathrm{N}\left(\mu, \sigma^{2}\right)$ que aceptamos en la práctica como la apropiada para la mayoría de las características de un producto industrial. El conjunto de valores que puede darse bajo la curva son representados por un valor característico que es cuantil de un determinado tanto por 100. En el ejemplo el $5 \%$.

En la Figura 4 la producción cumple estrictamente con el valor especificado al coincidir el valor característico fc,real y el valor especificado fck

La Figura 5 muestra la relación entre la curva de distribución de la características del producto y la distribución del criterio de aceptación. Se trata de un producto estricto, pues el valor característico del producto es igual al valor especificado. Como se puede ver, cuando se ha definido un criterio de distribución, lo importante es la relación con el valor especificado. En este caso, el valor especificado es un cuantil del $5 \%$ por la cola derecha. Al tratarse de un producto estricto el área que 
queda a la derecha del valor especificado fck es la denominada Riesgo del Consumidor. El criterio de aceptación se concreta con un valor de $\mathrm{K}=4$. Este valor es proporcionado por el simulador de Monte Carlo una vez fijado el Riesgo del Consumidor en 0,05 .

La Figura 6 muestra un caso semejante al de la Figura 5 con un valor de $K=1,019$, que es el que resulta cuando, siendo el producto estricto, el Riesgo del Consumidor deseado es $\beta=0,50$.

La Figura 7 (pág. siguiente) muestra el efecto de buscar un Riesgo del Consumidor $\beta=0,70$, que naturalmente es poco habitual, presentándose exclusivamente para mostrar el efecto del coeficiente $\mathrm{K}$ en el criterio de aceptación.

La Figura 8 (pág. siguiente) muestra cómo para un producto estricto la probabilidad de aceptación $\mathrm{P}(\mathrm{A})$ se reduce cuando el producto tiene un valor característico $f_{c}$ real inferior al especificado $f_{c k}$. Tanto en este caso como en el de la Figura 9, el criterio de aceptación es el mismo, con un valor de $\mathrm{K}=1,019$.

En la Figura 9 (pág. siguiente) la probabilidad de aceptación sube al 98\% debido a que el valor característico $\mathrm{f}_{\mathrm{c} \text {, real }}$ es mayor que el valor especificado $f_{c k}$.

La Figura 10 (pág. siguiente) representa las tres curvas $\mathrm{CO}$ que genera el criterio de aceptación genérico: $\bar{x}_{N}-K \cdot r_{N} \geq f_{c k}$ para los distintos valores de $\mathrm{K}$ expresados en las ecuaciones 18,19 y 20 :

$\bar{x}_{N}-0,720 \cdot r_{N} \geq f_{c k}$

$\bar{x}_{N}-1,019 \cdot r_{N} \geq f_{c k}$

$\bar{x}_{N}-4,000 \cdot r_{N} \geq f_{c k}$

También, podemos utilizar el simulador para comprobar qué probabilidad de aceptación tendría un supuesto producto fabricado por nosotros si se incumple el criterio de conformidad en un determinado grado o superamos el valor especificado sustancialmente. Obviamente esta comprobación se debe hacer con un valor de $\mathrm{K}$ resultante para el valor de $\beta$ pactado con anterioridad. De esta forma se comprueba la eficacia del criterio, es decir, su sensibilidad a los cambios de calidad del producto. Al comprador le interesará un criterio que sea poco sensible a las bajadas de calidad y, al suministrador, justamente, lo contrario. Como se puede comprobar para el mismo valor de $\mathrm{f}_{\mathrm{c} \text {,real }}=40$ unidades se corresponden tres valores distintos de $\beta$ cuando variamos el valor de K. Los valores de $\beta$ vie-
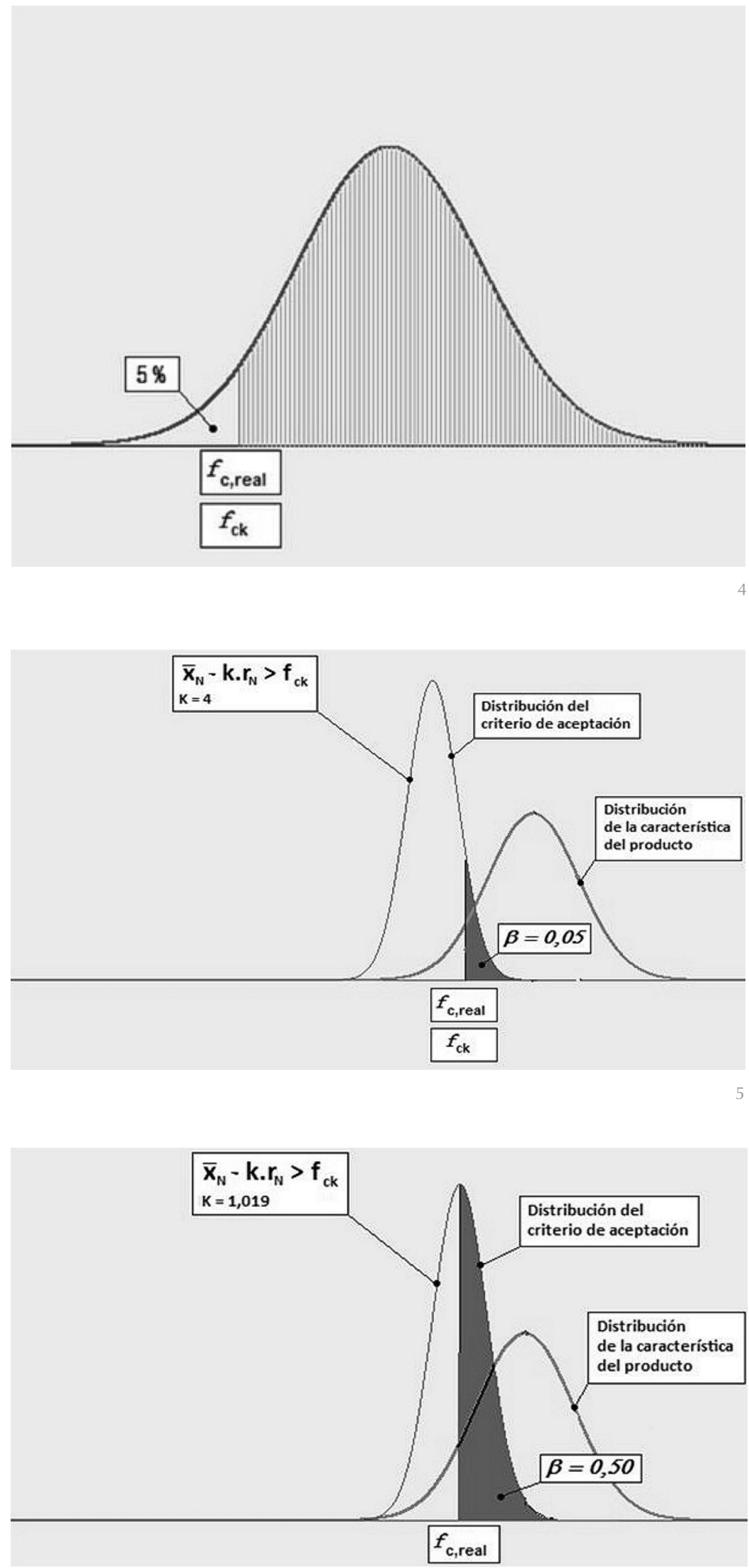

nen representados en las Figuras 4, 5 y 6 por el área a la derecha del valor especificado $f_{c k}$. Por otra parte, en la curva central, correspondiente a un valor de $K=1,019$, se representan

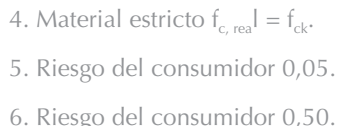

6. Riesgo del consumidor 0,50 . 

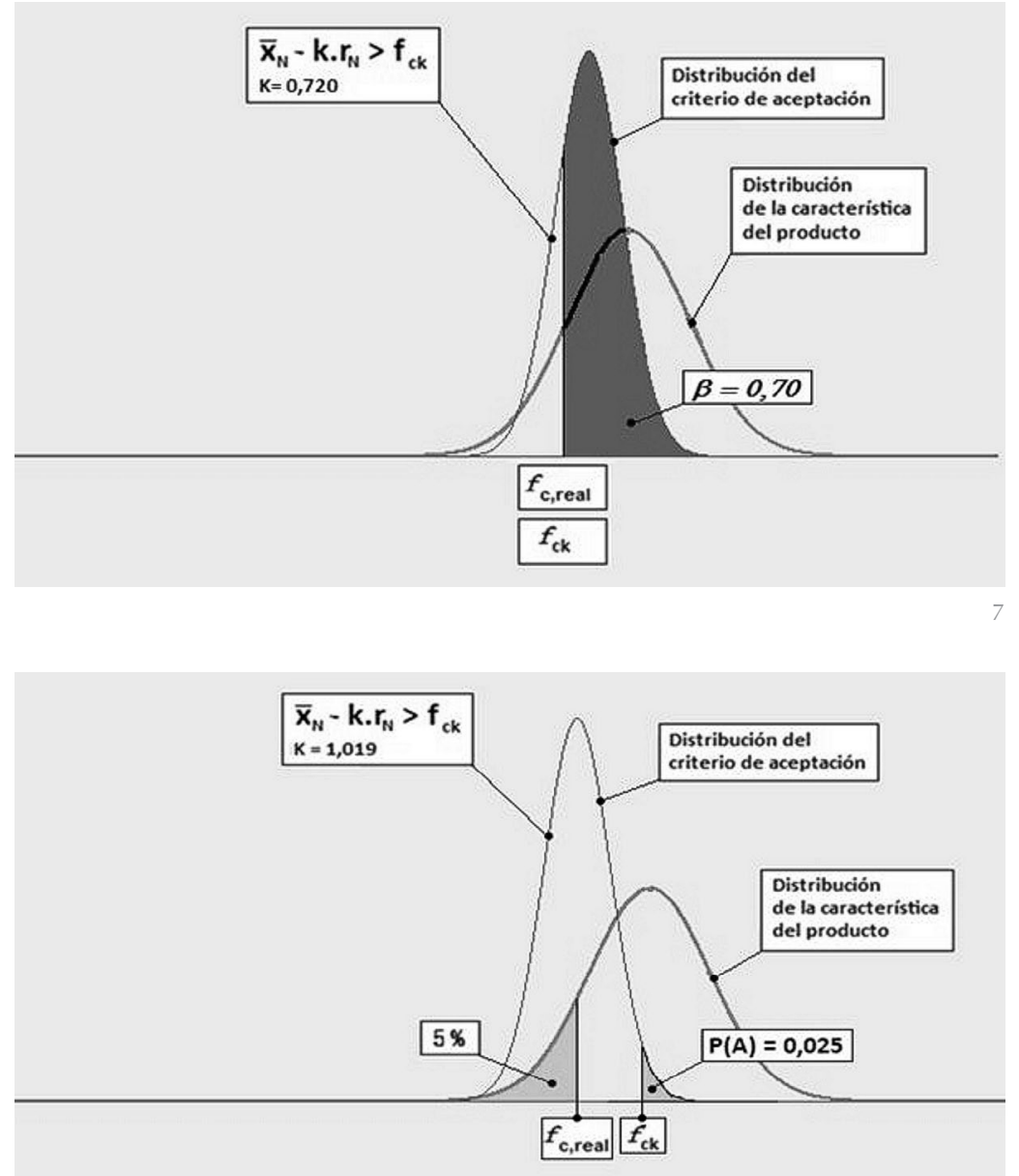

\section{Riesgo del consumidor 0,70.}

8. Probabilidad de aceptación 2,5\%.

9. Probabilidad de aceptación 98\%.

10. Curvas $\mathrm{CO}$ de distintos planes de control. dos puntos (cuadrados) señalados como 7 y 8 que representan las coordenadas 32,8-0,025 y 50,0-0,98 para dos situaciones del producto realmente fabricado. Finalmente mostramos en la Figura 11 el aspecto que presenta una hoja de cálculo EXCEL TM de Microsoft con el diseño del simulador.
Como se puede comprobar en la ventana operativa figura la fórmula generadora de los valores. Es el motor de la simulación. La fórmula del primer miembro del criterio de aceptación y de la comparación con el valor especificado que proporciona los $1 \mathrm{y}$ 0 permiten establecer la proporción (probabilidad) de aceptación en el simulador en la celda G50005 de la Figura 11.

\section{CONCLUSIONES}

Como es fácil de comprobar por aquellos que se dedican al control de calidad, en la construcción abundan las situaciones en las que no se cuenta con indicaciones sobre el control de calidad en los términos en que la estadística permite. Por otra parte, no siempre es posible que el criterio de aceptación elegido pueda ser tratado matemáticamente.

Con el simulador de Monte Carlo, sin embargo, se puede, con la ayuda de una hoja de cálculo que tenga las funciones estadísticas señaladas en este artículo, llevar a cabo un ajuste del criterio de aceptación suficientemente preciso y fundado en los intereses de las partes interesadas expresados por los riesgos aceptados de común acuerdo.

Por otro lado, con el simulador de Monte Carlo el suministrador está en condiciones de conocer qué margen debe establecer entre el valor especificado y el valor característico real de su producción para establecer una probabilidad de rechazo aceptable para sus intereses. En todo caso es una herramienta que hace transparente la relación mutua entre suministrador y consumidor en las relaciones de transferencia de productos.
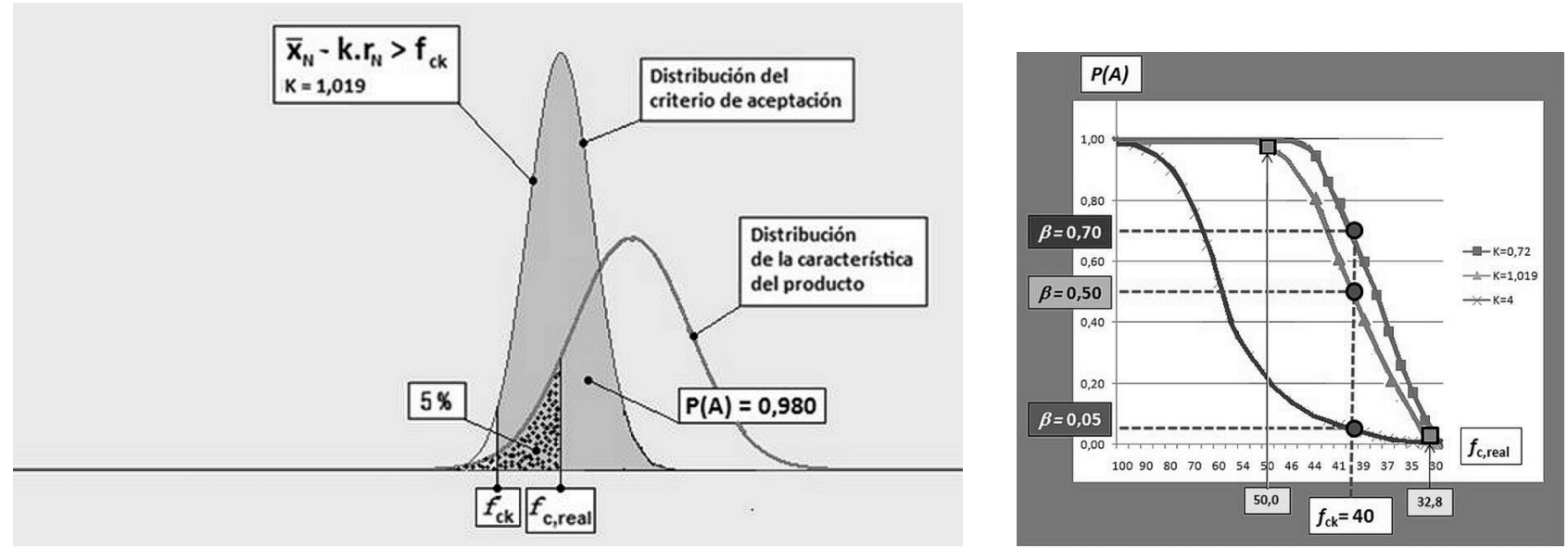


\begin{tabular}{|c|c|c|c|c|c|c|c|c|c|c|}
\hline \multicolumn{3}{|c|}{ AS } & 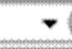 & \multicolumn{2}{|r|}{$f_{x}$} & \multicolumn{5}{|c|}{$=\$ A S 2+\$ B \$ 2^{*}$ DISTR.NORM.ESTAND.INV(ALEATORIO ()$)$} \\
\hline 4 & A & B & $\mathrm{C}$ & D & E & $\mathrm{F}$ & $G$ & $\mathrm{H}$ & 1 & $\mathrm{~J}$ \\
\hline 1 & $M$ & 0 & fck: & tcreal & $\mathrm{K}$ & & & & & \\
\hline 2 & 46,58 & 4 & 40 & 40 & 0,7200 & & & & & \\
\hline 3 & & & & & & & & & & \\
\hline 4 & $X_{1}$ & $x_{2}$ & $\mathrm{X} 3$ & $\mathrm{Xm}$ & $\mathrm{rN}$ & $\mathrm{Xm} \cdot \mathrm{K} \cdot \mathrm{rN}$ & AR & & & \\
\hline 5 & 50.7 & 44.3 & 37.8 & 44,3 & 12,9 & 35,01 & 0 & & & \\
\hline 6 & 42,1 & 43.1 & 46.6 & 43,9 & 4,5 & 40,71 & 1 & & & \\
\hline 7 & 48.1 & 50.6 & 46.3 & 48,3 & 4,3 & 45.23 & 1 & & & \\
\hline 8 & 50.4 & 39.2 & 48.9 & 46,2 & 11,1 & 38.14 & 0 & & & \\
\hline 9 & 44,2 & 42,1 & 50.2 & 45.5 & 8,0 & 39,73 & 0 & & & \\
\hline 10 & 48.0 & 49.7 & 47.7 & 48,5 & 2,0 & 47.05 & 1 & & & \\
\hline 11 & 45,1 & 44.2 & 48.7 & 46,0 & 4,5 & 42,80 & 1 & & & \\
\hline 12 & 44,3 & 55.2 & 44.8 & 48,1 & 10,9 & 40,21 & 1 & & & \\
\hline 13 & 45.9 & 40.5 & 49.7 & 45,4 & 9,2 & 38.79 & 0 & & & \\
\hline 14 & 54.6 & 51.7 & 47.4 & 51,2 & 7,2 & 46,01 & 1 & & & \\
\hline 15 & 50.7 & 48.9 & 44.1 & 47,9 & 6,6 & 43,15 & 1 & & & \\
\hline 16 & 48.9 & 51.1 & 52.3 & 50,8 & 3,4 & 48,32 & 1 & & & \\
\hline 17 & 49.7 & 41.9 & 45.4 & 45.7 & 7,8 & 40,06 & 1 & & & \\
\hline 18 & 44.8 & 44.2 & 43.8 & 44,3 & 1,0 & 43.52 & 1 & & & \\
\hline 50000 & 50.0 & 47.7 & 47.4 & 48,4 & 2,6 & 46.48 & 1 & & & \\
\hline 50001 & 52,9 & 42.9 & 47.6 & 47,8 & 10.0 & 40,61 & 1 & & & \\
\hline 50002 & 52,2 & 43,0 & 37.8 & 44,4 & 14,4 & 33.99 & 0 & & & \\
\hline 50003 & 51.2 & 46.1 & 44.5 & 47,3 & 6,7 & 42,48 & 1 & & & \\
\hline 50004 & 49.8 & 49.1 & 56.4 & 51.8 & 7,3 & 46.55 & 1 & & & \\
\hline 50005 & 46,6 & 46,6 & 46,6 & 46,6 & 6,7 & 41,74 & 0,705 & & & \\
\hline 50006 & 4.0 & 4.0 & 4.0 & 2,3 & & & & & & \\
\hline 50007 & & & & & & & & & & \\
\hline
\end{tabular}

\section{BIBLIOGRAFÍA}

(1) AENOR. Norma UNE 66020-1:2001 Procedimientos de muestreo para la inspección por atributos. Parte 1: Planes de muestreo para las inspecciones lote por lote, tabulados según el nivel de calidad aceptable (NCA). AENOR, 200

(2) AENOR. Norma UNE 66020-1:2002 ERRATUM. Procedimientos de muestreo para la inspección por atributos. Parte 1: Planes de muestreo para las inspecciones lote por lote, tabulados según el nivel de calidad aceptable (NCA).

(3) AENOR. Norma UNE 66030:1984, Reglas y tablas de muestreo para la inspección por variables de los porcentajes de unidades defectuosas. AENOR, 1984.

(4) AENOR. Norma UNE-ISO 3534-1. Estadística. Vocabulario y símbolos. Términos estadísticos generales y términos empleados en el cálculo de probabilidades. AENOR, 2008.

(5) AENOR. Norma UNE-ISO 3534-2. Estadística. Vocabulario y símbolos. Estadística aplicada. AENOR, 2008.

(6) Comisión Permanente Interministerial del Hormigón Estructural. Instrucción Española de Hormigón Estructural. CPH, Madrid, 2008.

(7) Garrido, A. et al., La estadística aplicada a la seguridad y la durabilidad del hormigón estructural. Congreso de ACHE 2003.

(8) Grant, E.L., Control Estadístico de Calidad, Compañía Editorial Continental S.A., México 1999.

(9) Kreyszig, E., Introducción a la Estadística Matemática, Editorial Limusa, México 1973.

(10) López Agüí, J.C. et al., Control Estadístico del Hormigón Estructural, ACHE, Madrid 2003.

(11) López Agüí, J.C., Fundamentos científicos del control de calidad óptimo del cemento (tesis inédita), UPM, Madrid 2002.

(12) López Aguí, J.C., Guía Básica para la simulación de Monte Carlo, AENOR, Madrid 2008.

(13) Young, H., Guía Completa Office2000, Mc Graw Hill, Madrid 1999. 